\title{
The Water Content of the Ureilite Parent Body
}

\author{
LIAM PETERSON ${ }^{1}$, MEGAN E. NEWCOMBE ${ }^{1}$, SUNE \\ GRØNLUND NIELSEN ${ }^{2}$, JIANHUA WANG ${ }^{3}$, ADAM R. \\ SARAFIAN $^{4}$ AND CONEL M. O'D. ALEXANDER ${ }^{3}$
}

\author{
${ }^{1}$ University of Maryland \\ ${ }^{2}$ Woods Hole Oceanographic Institution \\ ${ }^{3}$ Carnegie Institution for Science \\ ${ }^{4}$ Corning Incorporated \\ Presenting Author: 1dpete@umd.edu
}

The source(s), abundance, and timing of delivery of volatiles to the inner solar system are debated. Measurements of water in angrites [1], eucrites [2], and enstatite [3], ordinary [4], and Rumuruti chondrites [5] suggest that water was present in the inner solar system within the first $\sim 2$ Ma of solar system history, and therefore during Earth's primary accretion window; however the origin of this water is unclear. One source of uncertainty is the degree of desiccation due to melting and thermal metamorphism experienced by early-formed planetesimals. To evaluate the extent to which volatiles are retained during early accretion and melting, we can study the primitive achondrites, which are sourced from parent bodies that incompletely melted and differentiated, and retain some chemical, isotopic, and/or textural similarities with their chondritic precursors.

The ureilites are a group of C-rich, primitive achondrites from a single, early-formed parent body [6-7]. Following the NC-CC classification of meteorites, the ureilites have a $\mathrm{NC}$ affinity, represent a terrestrial end-member composition [8], and may represent a terrestrial precursor composition [9]. Despite the significance of the ureilites, there has been no systematic study of ureilite volatile contents.

We analyzed the $\mathrm{H}$ content of ureilite nominally anhydrous minerals (NAMs). Preliminary results suggest ureilite NAMs contain $\leq 9$ ppm $\mathrm{H}_{2} \mathrm{O}$, at the low end of the ranges of $\mathrm{H}_{2} \mathrm{O}$ concentrations reported for NAMs in angrites (6-21 ppm) [1] and eucrites $(4-18 \mathrm{ppm})[2,10]$. The relatively low volatile content of NAMs in the ureilite parent body may be influenced by magmatic degassing during melting, silicate smelting, impact(s), or thermal metamorphism on the parent body. Further analyses of ureilite refractory, moderately volatile, and volatile elements will be used to examine the potential for volatile delivery by CI chondrite like material.

[1] Sarafian et al. 2017, GCA; [2] Sarafian et al. 2019, GCA; [3] Piani et al. 2020, Science; [4] Alexander 2019, GCA; [5] McCanta et al. 2008, GCA; [6] Budde et al. 2015, EPSL; [7] Goodrich et al. 2015, MPS; [8] Warren 2011, EPSL; [9] Schiller et al. 2018, Nature; [10] Stephant et al. 2021, GCA 